\title{
Deep Breathing Alters Bistable Auditory Perception
}

\author{
Ahmad Yousef ${ }^{1}$ \\ ${ }^{1}$ School of Computational Science and Engineering, McMaster University, Hamilton, Ontario, Canada \\ *Correspondence: mohamas2@mcmaster.ca
}

\begin{abstract}
This article is to provide evidence that deep breathing can alters auditory bistability (verbal transformations). We had noticed that deep inhalation can significantly stop the auditory rivalry of the verbal transformation, namely, hearing the original material but not the illusive ones. This might be because the possible forcible reduction in the amount of oxygenated hemoglobin in the brain during the intended deep inhalation; in turn, different parts in the neocortex might be partially deactivated preventing the brain from have perceptual rivalrous conscious awareness. Deep exhalation, however, seemingly produce illusive perception that are sometimes relevant but temporally shifted to the original material, and other times partially irrelevant to the original material possibly because of abandoned oxygen-rich blood returns back to the brain allowing it to overwork and perceive the playback speed much faster, and thus, inter-stimulus interference.

Introduction

Respiratory sinus arrhythmia governs
\end{abstract} the breathing cycles; namely the heart rate raises for the inhalation and declines for exhalation, see references 1 and 2 . Deep inhalation and exhalation, however, can be volitionally controlled, and these neurophysiological voluntary processes can alter the visual motion perception, see reference 3 . In this study, however, we will study whether diaphragmatic breathing can alter the dynamics of the bistable auditory perception. We used a famous example of auditory bistability (verbal transformations) that had been discovered by Warren and Gregory back to 1958. They claimed that after prolonged exposure of unstoppable repetition of word 'life', most listeners reported subjective alternations between the original material and some transformed speech forms, namely the subject's auditory perception switches between 'life life life' and 'fly fly fly'. In their design, to comprehend the illusion, it was unclear whether the repeated cyclic word that exclusively contains the word 'life' in our research should have period of silence. To test whether the brain is able to achieve perceptual filling-in, see reference 5; and whether there is compression of the period of our auditory signal during exhalation; exhalation that's assumed to activate the peripheral conscious brain, see reference 3; we installed long silence periods, before and after the word 'life' as shown in the central infographic; and we found prefect rivalry is perceived. The central infographic the elementary cycle that should be repeated over and over to build our auditory stimulus. Important to mention, in 2007, two research groups had confirmed that the dynamics of verbal transformations were similar to other examples of bistable stimuli, see reference 6 , and 7; allowing us to claim that our present stimulus is an outstanding form of bistable auditory rivalry. Through this stimulus, and along with our previous study, see reference 8 , we hope to add more information to facilitate the distinction between central \& peripheral conscious brains for the human auditory awareness. Preliminary Installation \& Understanding the Variabilities Twelve human subjects were recruited to participate in this study. Human subjects with averaged size pupil have stable perceptual rivalry between life, and fly during their deep breathing, with mostly exclusive perception of word 'life' during deep inhalation, and word 'fly' during deep exhalation. Human subjects with dilated pupils had been also having
ability to hear the word 'life' when extremely high probability to hear the word 'life' wh
they perform deep inhalation, through their deep exhalation, however, in addition to word 'fly'; some of the human subjects had reported perception of the following words such as, life, night and flight. The original material should produce only perception of life and fly, but the interstimulus interference seems to overlap in a way that causes misperceptions. To visualize these kinds of interstimulus interference, readers are advised to view the visual stimulus offered in reference 3, namely, the illusory motion reversals, sometimes two cyclic elements are overlapped over each other but not perfect spatial overlapping, rather than with little spatial separation. Because of the aforementioned issues \& given that there is a common agreement about the effect of deep inhalation among all of the subjects, namely they have outstanding perception of the word 'life'; we had requests from all of the human subjects to report only the perception of 'life' 
word, but not the other perceptions. We therefore can know the deep inhalation can influence the auditory bistability. Noticeably, deep inhalation in visual perception slow the speed down, and it does the same here, thus perfect acquisition by the human hearing system to the original material.

\section{Materials \& Methods}

The stimulus consists of repeated cyclic elements which had been described and visualize in page1. The stimulus had been played back through loudspeaker; and

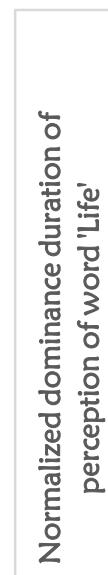

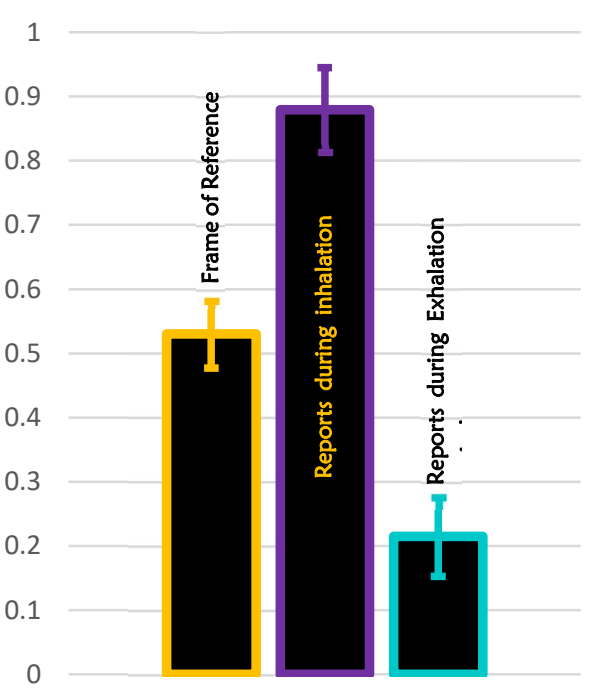

brain to over work, and thus, perception of irrelevant words discussed in the previous section.

Essential Notifications

We noticed that it is not important to passively hear the stimulus to experience the rivalry; the readers may be able to experience the rivalry by pronouncing the word 'life' quietly to themselves; however, simultaneous deep breathing would be impossible. We would like to emphasize that this stimulus mostly follows up the same each human subject controls the volume to ensure comfortable hearing. The experiment stages are as follow, presenting the frame of reference for twenty seconds to allow the human subjects to comprehend the auditory bistability, and to make that all of them is able to realize stable perceptual rivalry between word 'life' versus 'fly'. Afterwards, human subjects has to follow the same 'deep breathing' protocol offered in reference 3 . Each subject has to undergo the same experiment, see reference 8 , for three times to ensure fine-tuned behavioral data. Each human subject has to report when the perception of word 'life' in the frame of reference, deep inhalation and deep exhalation by pressing key 1 . The behavioral data were collected from the three identical trials, and then compared with the original material (the actual number of the cyclic elements), and the outcome values were afterwards normalized. The three trials values are averaged for each subject separately. The aforementioned mean values of all of the twelve subjects are used to estimate simplest statistical values, namely, the average and the standard error of the mean; values that determine the relativeness between the reality and the perception.

Results and Conclusion

As shown in the up-central infographic, deep inhalation and deep exhalation have great influence on the auditory bistability. In reference 3, it was clear that deep inhalation reduces the perceived speed, and we have the same effect here; in fact, that reduction in the perceived speed disallow the perception to have what we call inter-'cyclic element' interference. Deep exhalation on the other hand increases the perceived speed, allowing the perception to have the aforementioned interference, therefore, not only the perception of fly, which is nothing but a derivative of life $\rightarrow$ 'f-lie'; but the possible increased amount of oxygenated hemoglobin red blood cells might trigger the role offered in reference 9. Namely, the visual awareness represented by the face recognition, is a result of temporal hierarchical process; and the last signal screened \& acquired by the sensory receptors is responsible for the final decisive awareness. We therefore think the temporal readiness of the sensory receptors plays a role in the perceptual rivalry in general. We have to emphasize that deep breathing seems to be the governor of the auditory bistability.

We would like to ensure that readers should have direct access to the stimuli, see reference 10. Advisably, download the experiment for the best quality. We wish to collaborate with scholars with brain imaging, ECG, and professional eye-tracker facilities in challenging investigations. Enquiries may be sent to the author. Transactional References

[1] Berntson etal., (1993). Respiratory sinus arrhythmia: autonomic origins, physiological mechanisms, and psycho-physiological implications. Psychophysiology.

[2] C. Ludwig, (1847). On the influence of respiratory movements on blood flow in the aortic system. Leipzig.

[3] Yousef, A. 2019. Deep Breathing Alters Visual Motion Perception. PsyArXiv. doi:10.31234/osf.io/up3sa.

[4] Warren, and Gregory (1958). An auditory analogue of the visual reversible figure. Am. J. Psychol.

[5] Ikuya Murakami, 2009. Perceptual Filling-In. Encyclopedia of Neuroscience.

[6] Sato, et al. (2007). Visual contribution to the multistable perception of speech. Percept. Psychophys.

[7] Kondo, and Kashino (2007). Neural mechanisms of auditory awareness underlying verbal transformations. Neuroimage.

[8] Yousef, A. 2019. Distinctions Between Central and Peripheral Hearing. PsyArXiv. doi:10.31234/osf.io/ps2mh.

[9] Yousef, Ahmad. 2019. Two Distinct Fusiform Face Areas. PsyArXiv. doi:10.31234/osf.io/a8gzv.

[10] You may find the stimuli in the following link: https://drive.google.com/drive/folders/1aFjOqqQ5r6NslhRVOqohJbl VsxcqRDAV 